\title{
Multivaluedness in Networks: Theory
}

\author{
Michaël Anton(ie) van Wyk Member, IEEE
}

\begin{abstract}
This brief note reports the fundamental phenomenon of implicit multivaluedness exhibited from one output to the other of two node-systems with a common inputreferred to as counter-cascaded ${ }^{1}$ systems-under the appropriate conditions. The novel concepts of immanence and transcendence are introduced upon which the formulation and prove of a necessary and sufficient condition for multivaluedness are based; this is the main result of this note. Next, subsequent consequences of this result are presented. Among these is the fact that this result also holds for cascaded generalized systems.

The novel application of structural complexity reduction in directed networks presented next, demonstrates the utility of multivaluedness and is itself a contribution to the theory of signals and systems.

The significance of the work presented here is that it contributes toward the theory of systems and networks as well as toward the arsenal of tools for studying networks.

Index Terms-Big data, cascaded systems, complex networks, counter-cascaded systems, distributed measurement systems, functional uniformization, immanence, mixed modeling, multivaluedness, multivalued function, multivalued relation, network analysis, network science, networked systems, neural networks, node rationalization, nonlinear systems, structural reduction, transcendence, single-valuedness, single-valued relation, welldefined mapping.
\end{abstract}

\section{INTRODUCTION}

The area of Network Theory and Analysis [1] has rapidly expanded into a very active and vibrant field of research, with ever more fundamental theoretical results [2] and novel applications [3] [4] being reported. A network consists of units called nodes that interact via connections referred to as links or edges. In order to give a glimpse of the diverse nature of these objects of study, note that size-wise, real-world networks range from a few nodes up to billions of nodes and beyond, with some node-pairs interacting unilaterally (via directed edges) and others bilaterally (via undirected edges). Structure-wise, they range from highly homogeneously structured networks through to amorphously unstructured and even randomly structured networks. Character-wise, they vary

Financial Support Acknowledgment: This work was supported in part by the Carl and Emily Fuchs Foundation's Chair in Systems and Control Engineering at the University of the Witwatersrand, Johannesburg, South Africa and by a grant from the Innovation and Technology Funding (ITF) of the Hong Kong Special Administrative Region, China [ITS/359/17].

Michaël A. van Wyk is with the School of Electrical and Information Engineering, The University of the Witwatersrand, Johannesburg, South Africa and City University of Hong Kong, Hong Kong SAR, China (e-mail: anton.vanwyk@wits.ac.za, mavanwyk@gmail.com).

\footnotetext{
${ }^{1}$ In Fig. 1 the systems $T$ and $M$ are counter-cascaded systems with common input $u$, considering outputs $v$ and $w$; similarly are $N \circ T$ and $M$, considering outputs $x$ and $w$.
}

from uniformly cooperative or competitive to heterogeneously mixed with cooperative and competitive factions contained within. Furthermore, the mathematical descriptions of nodes in a network range from uniform (identical) in some networks, to diverse (distinct) in others. For these reasons, graph-theoretic methods are indispensable for the description and analysis of network problems. An important feature of the majority of real-world networks is that they are constantly evolving as far as structure, character and mathematical description are concerned.

In the literature, the meaning of the term "network analysis" is rather diverse. Of particular interest here, is the extended definition of Zaidi [5], namely that it encapsulates the study of theory, methods and algorithms applicable to graph-based models representing interconnected real-world systems. From this perspective, the collection of interconnected elements of a finite element analysis of a distributed structure or physical field and a complex interconnection of nonlinear dynamical systems are instances of network analyses [6], [7], the former undirected and the latter directed. Both an excellent account of the theory and overview of current research directions in networks, can be found in [8] and [9].

Even though networks might not always have external causes (inputs) and effects (outputs), there are always internal (i.e., local) causes and effects of interest when considering a single node or a collection of nodes. A deeper understanding of the global behavior and dynamics of a network usually requires a deeper understanding of the mechanisms of behavior at a more detailed level in the network. For this reason, oftentimes it requires one to relate two (sets of) effects $w \in W$ and $x \in X$, produced by the common cause $u \in U$ (as in Fig. 1), in order to gain deeper insight. This aspect, termed counter-cascaded systems, which is ubiquitous in directed networks and systems, is the focus of this note, with results for cascaded systems following as a byproduct. In particular, minimal underlying assumptions and an elementary set-theoretic argument [10], [11], produce a necessary and sufficient condition for such a relation to be well-defined.

The outline of this paper is as follows: Section II presents the mathematical foundations: basic assumptions, definitions, the main result, its proof and some immediate consequences. The application considered in Section III demonstrates the utility of these results in structural reductions of directed networks, referred to as functional uniformization via node rationalization. The conclusion follows in Section IV.

\section{Theory OF Multivaluedness}

In order to provide a definite and concrete context for the presentation and discussion, we consider (directed) networks 


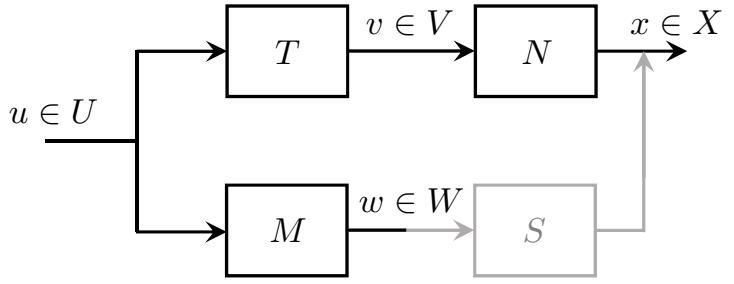

Fig. 1. Two counter-cascaded paths with the common cause, $u \in U$. The implicit relation $S$ is shown in gray.

of nonlinear dynamical systems ${ }^{2}$ [12]. In such networks, we will study occurrences of counter-cascaded systems using the framework shown in Fig. 1. Importantly, note that this framework includes both counter-cascaded $(M \neq I)$ and cascaded $(M=I)$ systems configurations; $I$ denotes the identity mapping. Therefore, the results obtained here are applicable to both of these configurations. In general $U$, $V, W$ and $X$ can be very general sets, with $M \subset U \times W$, $T \subset U \times V$ and $N \subset V \times X$ binary relations. However, here these relations are mappings $M: U \rightarrow W, T: U \rightarrow V$ and $N: V \rightarrow X$. For the selected context, unless stated otherwise, these mappings are nonlinear operators ${ }^{3}$ with domains and ranges real vector spaces; typically $T$ is a nonlinear operator describing some nonlinear system, with $M$ and $N$ nonlinear operators describing either nonlinear systems, input and output ancillary systems or even identity operators. When they describe ancillary systems, $M$ and $N$ are often many-toone mappings as is inevitably the case when they represent measurement systems.

For the purpose of the presentation here, $N$ is redundant and can be absorbed into $T$ by replacing $N \circ T$ with $T$ or by setting $N=I .{ }^{4}$ However, for applications of these results in other areas, it has a distinct and explicit purpose, as will be reported on in the future. Finally, the implicit relation $S \subset W \times X$ will either be single-valued (i.e., a mapping) or multivalued, depending on the problem considered.

Next follows an important definition:

Definition II.1. (Immanence, Transcendence) In Fig. 1, the mapping $T$ is called immanent with respect (or relative) to the ordered pair of mappings $(M, N)$, if for every element $w \in M(U)$, there exists an element $x \in N(T(U))$ such that $T\left(M^{-1}(w)\right) \subseteq N^{-1}(x)$.

If not immanent with respect to $(M, N)$, then $T$ is called transcendent with respect to $(M, N)$.

\section{Notes.}

\footnotetext{
${ }^{2}$ For economy of presentation, throughout, the same symbol is used both for a system and its mathematical model; these are usually not the same.

${ }^{3}$ In order to emphasize that the results presented here apply in much more general contexts, the term "mapping" will be used instead of "operator."

${ }^{4}$ Throughout, $N \circ T$ denotes function composition $N(T(\cdot))$ of $N$ with $T$ and $M^{-1}$ denotes the preimage of $M$.

${ }^{5}$ If such $x$ exists, then it is unique. To see this suppose that two such elements $x_{1}$ and $x_{2}$ exist, implying that $N^{-1}\left(x_{1}\right) \cap N^{-1}\left(x_{2}\right) \neq \emptyset$. Now, applying $N$ to this nonempty intersection immediately yields $x_{1}=x_{2}$.
}

a. The statement " $T$ is $(M, N)$-immanent" is often used to mean " $T$ is immanent with respect to $(M, N)$ " and similarly for transcendence.

b. Two nodes in a network, can be analyzed for immanence or transcendence only if they are affected by the very same cause.

c. Since collections of nodes can be clustered to form supernodes, which are themselves nodes, this definition and all subsequent results apply to supernodes without explicit further mention.

The main result and its proof follows next:

Theorem II.2. (Well-Defined Mapping) The mapping $T$ is immanent relative to $(M, N)$ if and only if $N \circ T \circ M^{-1}$ is well-defined (i.e., single-valued).

Note. Before proceeding with the proof, first observe that for each element $u \in U$, there exist elements $w_{u}:=M(u)$ and $x_{u}:=N(T(u))$. Next, we associate $w_{u}$ and $x_{u}$ by writing $x_{u}=S\left(w_{u}\right)$ for every $u \in U$. This can be compactly expressed as $S:=N \circ T \circ M^{-1}$. Here, $S$ defines a relation. If for every pair of distinct elements $u_{1}, u_{2} \in U$ we have that $w_{u_{1}}=w_{u_{2}}$ implies that $x_{u_{1}}=x_{u_{2}}$, then $S$ is well-defined.

Proof. We first prove the "only if" part. Suppose that $T$ is immanent with respect to $(M, N)$. Now, if $S$ is not welldefined, then there exist distinct elements $u, u^{\prime} \in U$ such that $M(u)=M\left(u^{\prime}\right)$ but $x:=N(T(u)) \neq N\left(T\left(u^{\prime}\right)\right)=: x^{\prime}$. This contradicts the consequence of immanence, namely that $N(T(u))=x$ for all $u \in M^{-1}(w)$ and consequently $S$ is well-defined.

Conversely, to prove the "if" part, suppose $T$ is transcendent with respect to $(M, N)$. Then, for some $w \in M(U)$, there are distinct elements $u, u^{\prime} \in M^{-1}(w)$ for which $x:=N(T(u)) \neq$ $N\left(T\left(u^{\prime}\right)\right)=: x^{\prime}$, implying that $S$ is not well-defined because $S(w)=x$ and $S(w)=x^{\prime}$ and yet $x \neq x^{\prime}$. This concludes the converse via the contrapositive and completes the proof.

An equivalent statement of this result follows:

Theorem II.3. (Multivalued Relation) The mapping $T$ is transcendent relative to $(M, N)$ if and only if $N \circ T \circ M^{-1}$ is multivalued (i.e., not well-defined).

To our knowledge this result, identifying all those situations when the outputs of two counter-cascaded subsystems are functionally related (as well as when not), is a novel result.

Some immediate consequences of Theorem II.2 now follow.

Corollary II.4. (Existence of a Unique Faithful Model) For a given mapping $T$, a unique faithful model or modeling mapping $S$ exists if and only if $T$ is $(M, N)$-immanent.

For modeling problems, if $T$ is $(M, N)$-immanent, then there exists a unique mapping $w \mapsto S(w)$ which yields a unique faithful model of $T$, as perceived through $M$ and $N$, namely, $S(w)=N \circ T \circ M^{-1}(w)$ for every $w \in W$. In this 
case, $T$ can be "fully explained" by the combined abilities of the pair $(M, N)$ justifying the use of the term "immanence" used here. On the other hand, if $T$ is $(M, N)$-transcendent, then $S=N \circ T \circ M^{-1}$ is a (multivalued) relation which cannot be described by any mapping, whatsoever, and hence no faithful model exist; a "full explanation" of $T$ is beyond the combined abilities of the pair $(M, N)$, justifying use of the term "transcendence."

Still concerning modeling problems, approximation of $S$ becomes essential in the following situations: either $T$ is $(M, N)$-transcendent, or $T$ is $(M, N)$-immanent but the solution $N \circ T \circ M^{-1}$ is excluded from the allowable set of candidate model mappings, based on prior considerations. In these situations, the only remaining course of action is to select a qualifying approximation $\hat{S}_{o p t}: W \rightarrow X$ that approximates $S$ optimally according to some nonnegativevalued optimality criterion $J(e)$, with the error-relation $e$ defined by $X \supseteq e(t, \cdot):=S(t, \cdot)-\hat{S}(t)$, for all time $t^{6}$

Corollary II.5. Let $M, T, N$ and $S$ be as depicted in Fig. 1 .

a. If $M$ is given and $T$ is of canonical form $T=F \circ M$ for some fixed $F: W \rightarrow V$, then $T$ is $(M, N)$-immanent for every $N$.

b. If $M$ is injective then every $T$ is $(M, N)$-immanent for every $N$.

c. If $M$ is many-to-one, $T$ is not of canonical form and $N$ is one-to-one, then $T$ is $(M, N)$-transcendent.

d. If the condition for $(M, N)$-immanence holds everywhere in $B \subseteq U$, then $T \mid B$ is $(M \mid B, N)$-immanent. ${ }^{7}$

e. If $M$ is one-to-one, $N \circ T$ is many-to-one and $T$ is $(M, N)$-immanent, then the mapping $S$ is many-to-one.

f. If mappings $M, N$ and $S$ are given and there exists a $T$ satisfying the identity $N \circ T=S \circ M$, then $T$ is $(M, N)$ immanent. As a candidate solution, $N^{-1} \circ S \circ M$ is a well-defined mapping if and only if $N$ is injective.

g. If $M=I$ then a necessary and sufficient condition for the relation ${ }^{8} T \subset U \times V$ to be a mapping is that $T$ is $(I, N)$-immanent.

Notes.

a. If $M$ is many-to-one and $T$ is not of the canonical form $T=F \circ M$, then the $(M, N)$-immanence of $T$ depends on the choice of $N$.

b. In Corollary II.5(f), if $N$ is injective, then the expression $N^{-1} \circ S \circ M$ gives an explicit formula for $T$. However, if $N$ is not injective then $T$ satisfies the expression $N^{-1} \circ N \circ T=N^{-1} \circ S \circ M$ which is generally not solvable for $T$ since $N^{-1} \circ N \neq I$. So, unless additional information about $T$ is available, we can merely test candidate mappings $T$ to determine if they satisfy this expression; if one does, it follows immediately that $T$ is $(M, N)$-immanent.

\footnotetext{
${ }^{6}$ When $S$ is multivalued, then the difference in the expression for $e(t, \cdot)$ refers to the value $\hat{S}(t)$ subtracted from every element in the set $S(t, \cdot)$. When $S$ is single-valued, $e(t, \cdot) \equiv e(t)$ is a real number for each time instant $t$.

${ }^{7}$ Here, $T \mid B$ is the restriction of $T$ to the subset $B$ of its domain $U$.

${ }^{8}$ Such a relation $T$ can be interpreted as a generalized system.
}

Now, a little thought reveals the following to be true for configurations similar to that shown in Fig. 1, but with additional exogenous causes entering:

Lemma II.6. (Resolution of Exogenous Causes) Suppose that, along one of the paths of a counter-cased pair, another cause enters. Proceed to adjoin this cause to the original cause and adjoin the identity operator to the up-stream (i.e., unaffected) portion of the affected path, both by direct-sum operations. After this transformation, the mappings $M$ and $T$ may now be defined as before.

This is best demonstrated by the simple example consisting of two cascaded systems $\widetilde{v}=T_{1}(u)$ and $v=T_{2}(\widetilde{u}, \widetilde{v})$ with $\widetilde{u}$ the exogenous cause entering. Lemma II.6 states that, algebraically $\widetilde{u}$ can be referred toward the "root cause" $u$ as follows,

$$
v=T_{2}\left(\widetilde{u}, T_{1}(u)\right)=T_{2}\left(\widetilde{I}(\widetilde{u}), T_{1}(u)\right) \equiv \overbrace{T_{2} \circ\left(\widetilde{I} \oplus T_{1}\right)}^{T} \overbrace{(\widetilde{u}, u)}^{\mathbf{u}},
$$

with the direct-sum cause $(\widetilde{u}, u)$ feeding into the direct-sum system $\widetilde{I} \oplus T_{1}$ obtained by adjoining the identity mapping $\widetilde{I}$ to the up-stream unaffected portion $T_{1}$ of the affected path $T_{2}\left(\cdot, T_{1}(u)\right)$. The exogenous cause $\widetilde{u}$ has now been resolved yielding $v=T(\mathbf{u})$, the new system of interest.

In the case of counter-cascaded systems, there are two possible directions to be considered for immanence or transcendence. The following definition extends the previous definition to cover both these possibilities. For this, $N$ is removed by choosing it to be the identity mapping.

Definition II.7. (Bi-immanence, Bi-transcendence) In Fig. 1, if $T$ is $(M, I)$-immanent and $M$ is $(T, I)$-immanent, then $T$ and $M$ are called bilaterally immanent or bi-immanent. Similarly, if $T$ and $M$ are $(M, I)$-transcendent and $(T, I)$ transcendent, respectively, then $T$ and $M$ are called bilaterally transcendent or bi-transcendent.

Note.

Considering both directions for two counter-cascaded systems, in principle, all four of the following cases are possible: immanent-immanent (I-I), immanent-transcendent (I-T), transcendent-immanent (T-I) and transcendent-transcendent (T-T). For the case I-I the mapping relating the outputs is a bijection while, for the case T-T, there is no mapping that relates the two outputs in either direction. The case I-T implies that such a mapping exists in one direction but not in the other; vice versa for the case T-I.

\section{Application: Node Rationalization}

The results of the previous section will now be applied to structural reduction in directed networks in a novel manner. We start by introducing the necessary terminology. The process of expressing node mappings in factored form, with the rightmost factors chosen from as few as possible unique ones, is referred to as functional uniformization. Furthermore, the 


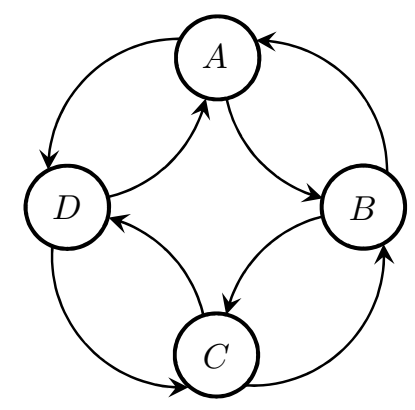

Fig. 2. A directed geographical network immediate neighbor interaction.

process of minimizing the number of nodes in a functionally uniformized network, by merging as many nodes as is possible to share common right-most factors and (node) inputs, is termed node rationalization. This form of structural transformation of a network results in a reduction in the number of nodes, with each of the resultant nodes possessing either multiple inputs or multiple outputs or both. The resulting network has fewer connected dynamical systems, represented by nodes, with vector (i.e., parallelized) edges joining them.

Consider the simple yet general four-node directed geographic network with immediate neighbor interaction, shown in Fig. 2. As before, the same symbol is used to present both the node and the mathematical model describing its behavior, i.e., its mathematical description. For example, $A$ identifies the upper-most node in Fig. 2; it also represents the mathematical mapping $A(\cdot, \cdot)$ that describes this node's behavior.

Unless additional information is available, no structural reduction of this network is possible. So, suppose that $C$ is $(A, I)$-immanent. Then, according to Corollary II.4, there exists a modeling mapping $\bar{C}$, as indicated in Fig. $3 .{ }^{9}$ Following Lemma II.6, we can adjoin the inputs feeding into nodes $A$ and $C$ to obtain a common vector input feeding into both $A$ and $C$, as depicted by the bold line in Fig. 4(a). Fig. 4(b) shows that the mapping $C$ can be replaced by the composition $\bar{C} \circ A$ as follows from Corollary II.4. This means that we can now replace node $C$ of the network with a "node" $\bar{C}$ which has a single input, fed by the output of node $A$ as shown in Fig. 5(a). The output of node $\bar{C}$ then replaces the output of node $C$, feeding into nodes $B$ and $D$ (Fig. 5(a)). To reduce this network to a three-node network requires us to merge $A$ and $\bar{C}$ into a single node with mathematical description $(I \oplus \bar{C}) \circ A$ yielding a vector output which feeds into nodes $B$ and $D$ via the bold edges in the graph shown in Fig. 5(b). The symbol $\oplus$ represents the direct sum operation.

Now, if there were no further immanence present in the network, then Fig. 5(b) shows the simplest network to which the original network can be structurally reduced, using node rationalization.

Next, in addition, assume that $D$ is $(B, I)$-immanent. Once again, by application of Lemma II.6 and Corollary II.4, there exists a modeling mapping $\bar{D}$ such that $D=\bar{D} \circ B$. Following the same procedure as above, the network can now be reduced

\footnotetext{
${ }^{9}$ In order to simplify matters, during the intermediate steps that follow, we relate back to earlier theoretical results by using the systems representation employed before.
}

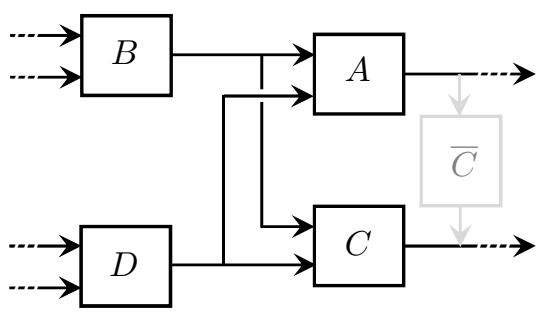

Fig. 3. Node $C$ is $(A, I)$-immanent with model $\bar{C}$.

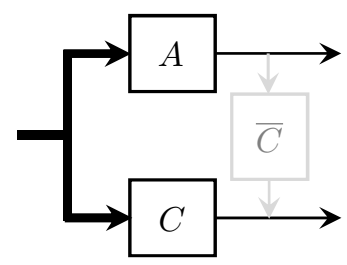

(a)

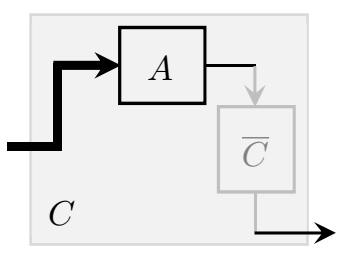

(b)
Fig. 4. Node $C$ is $(A, I)$-immanent with model $\bar{C}$. (a) Nodes $A$ and $C$ with the common vector input. (b) Node $C=\bar{C} \circ A$ by virtue of Corollary II.4.

to the form shown in Fig. 6(a)—effectively the two-node uniformized network shown in Fig. 6(b). To see this simply define the two nodes to have the mathematical descriptions ${ }^{10}$ $(I \oplus \bar{C}) \circ A$ and $(I \oplus \bar{D}) \circ B$, respectively, resulting in the interconnecting edges to become vector-valued. The final result consists of only two coupled systems. This reduction is striking, considering the generality of the mathematical descriptions of the four nodes of the original network.

To summarize, if our example network was completely void of immanence, i.e., all counter-cascaded node pairs were

\footnotetext{
${ }^{10}$ For economy of presentation and for readability, we represent the two identity mappings $I_{A}$ and $I_{B}$, operating on the ranges of $A$ and $B$, respectively, using the same symbol, namely $I$.
}

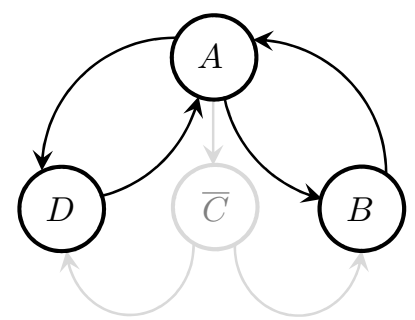

(a)

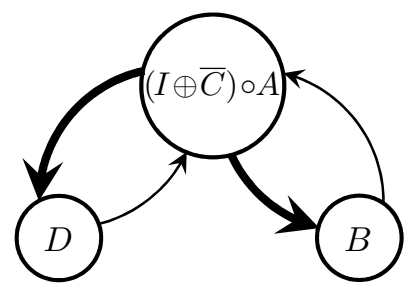

(b)
Fig. 5. Reduced network: (a) With node $C$ represented by $\bar{C} \circ A$ thus sharing the existing node $A$. (b) With the outputs of the new node consolidated.

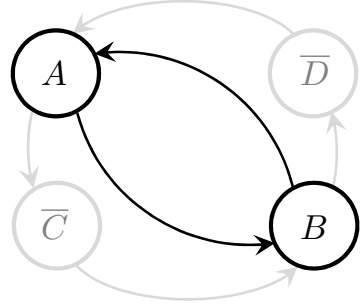

(a)

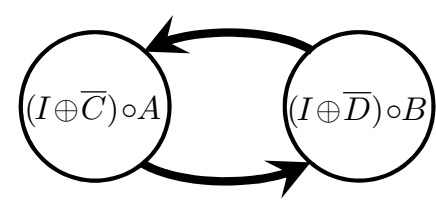

(b)
Fig. 6. Uniformized network: (a) With the explicit factorizations $\bar{C} \circ A$ and $\bar{D} \circ B$ explicitly shown. (b) With the outputs of the new nodes consolidated. 
transcendent, then by Theorem II.2, the original network would not in any way have been structurally reducible using node rationalization. In other words, except perhaps for cosmetic changes, Fig. 2 would then represent the simplest form possible for this network and imposing node rationalization in such a case, would yield unavoidable and unresolvable modeling errors, an inevitable consequence of transcendence.

To the other end, if bi-immanence was present, then options to interchange the roles of relevant nodes would have existed, while producing equivalent results.

In conclusion we point out that, in this example application, we stretched the presence of immanence to the limit in order to demonstrate the compactness of representation produced by the node rationalization. However, in real-world network investigations, node rationalization will usually only be applied selectively to expose important latent properties that would otherwise have gone unnoticed.

\section{CONCLUSion}

In this note, the fundamental phenomenon of multivaluedness in systems and networks was reported and studied. For this purpose, the novel notion of counter-cascaded configuration was first introduced. This, together with the new concepts of immanence and transcendence, facilitated the statement and proof of the key theoretical result in Theorem II.2, namely a necessary and sufficient condition for implicit multivaluedness to be exhibited by counter-cascaded nodes of a directed network. Subsequent corollaries provided further useful results for determining multivaluedness, given specific conditions. The configuration framework considered for this theoretical development, includes both the cases of counter-cascaded and cascaded node configurations which implies that these results can be applied to both configurations.

For the particular class of modeling problems considered by Corollary II.4, transcendence is an adverse characteristic. However, some work currently in progress suggests that transcendence is not necessarily always undesirable.

Next, a systems-and-signals type application, namely network uniformization via node rationalization, applied to a simple yet very general four-node directed geographic network, was presented. This demonstrated these results' potential to contribute toward the arsenal of tools for studying networks. As a fundamental phenomenon and its mathematical characterization, this work contributes toward the discipline of systems and networks science.

Further work is in progress to apply these theoretical results to distributed measurement systems and mixed modeling in networks, big data and neural networks and analysis of signal processing algorithms. Also underway, is work to extend these theoretical results to include the case of noise contamination in networks.

\section{ACKNOWLEDGMENT}

This work was financially supported by the Carl and Emily Fuchs Foundation through the research chair in System and
Control Engineering at the University of the Witwatersrand, Johannesburg, South Africa and by a grant from the Innovation and Technology Funding (ITF) of the Hong Kong Special Administrative Region, China [ITS/359/17] at the City University of Hong Kong, Hong Kong SAR, China.

The author wishes to thank A.M. McDonald, A.M. van Wyk and $\mathrm{G} . \mathrm{Ng}$ for proofreading the manuscript and for technical assistance during the preparation process.

\section{REFERENCES}

[1] M. Newman, Networks. 2nd ed. Oxford, UK: Oxford University Press, 2018.

[2] G. Minello, A. Torsello, E.R. Hancock, "Open system quantum thermodynamics of time-varying graphs," J. Complex Netw., vol. 8, no. 1 , Feb. 2020. [Online]. Available: https://academic.oup.com/comnet/article/ $8 / 1 /$ cnaa004/5741157

[3] A.G. Tennant, C.M.L. Smith and J.E. Chen C, "Who was the greatest of all-time? A historical analysis by a complex network of professional boxing," J. Complex Netw., vol. 8, no. 1, Feb. 2020. [Online]. Available: https://academic.oup.com/comnet/article/8/1/cnaa009/5770924

[4] Special Issue on Complex Networks Applications in Circuits and Systems, IEEE Circuits Syst. Mag., vol. 10, no. 3, Sept. 2010.

[5] F. Zaidi, "Analysis, Structure and Organization of Complex Networks," Ph.D. dissertation, Dept. Comput. Sci., The University of Bordeaux I, Bordeaux, France, 2010. [Online]. Available: https://tel.archivesouvertes.fr/tel-00542703

[6] L. Xiang, F. Chen, W. Ren, G. Chen, "Advances in Network Controllability," IEEE Circuits Syst. Mag. vol. 19, no. 2, pp. 8-32, Jun. 2019.

[7] C.W. Wu, "Evolution and Dynamics of Complex Networks of Coupled Systems," IEEE Circuits Syst. Mag. vol. 10, no. 3, pp. 55-63, Sept. 2010.

[8] G. Chen, X. Wang and X. Li, Fundamentals of Complex Networks: Models, Structures, and Dynamics. Singapore: John Wiley \& Sons, 2015.

[9] E. Estrada, The Structure of Complex Networks: Theory and Applications, Oxford, UK: Oxford University Press, 2011.

[10] H. Krim and A.B. Hamza, Geometric Methods in Signal and Image Analysis. Cambridge, UK: Cambridge University Press, 2015.

[11] R.A. Kennedy and P. Sadeghi. Hilbert Space Methods in Signal Processing. Cambridge, UK: Cambridge University Press, 2013.

[12] C.A. Desoer and M. Vidyasagar, Feedback Systems: Input-Output Properties. New York, USA: Academic Press, 1975. 